\title{
OSSERVAZIONI SU UNO SCAMBIO DI POEMETTI TRA PIETRO STANCOVICH E MARTINO FIORANTI
}

\author{
Sandro Cergna \\ Università Juraj Dobrila di Pola \\ Dipartimento di studi in lingua italiana
}

\section{Riassunto}

Il lavoro presenta un'analisi comparata tra due poemetti d'occasione in dialetto istroveneto dell'Istria sud-occidentale. I componimenti, scritti nel 1826 dal dignanese Martino Fioranti e dal canonico di Barbana Pietro Stancovich, rappresentano un'importante attestazione della presenza di un'attività poetica in quella che rappresentava all'epoca la koiné del territorio istriano, cioè l'idioma di diretta derivazione veneziana. I poemetti rivestono scarsa importanza a livello letterario. Più interessanti si rivelano, invece, se osservati da una prospettiva storica e documentaristica, attraverso la quale ci si svela l'ambiente, il costume dell'epoca, e soprattutto il rapporto di cordiale amicizia che univa i due poeti. In chiusura del lavoro si dà un breve confronto tra il poemetto e i tre sonetti di Martino Fioranti, rinvenuti nell'archivio della Biblioteca universitaria di Pola.

Parole chiave: poemetto, dialetto, istroveneto, Fioranti, Stancovich

1. Sull'esistenza di un cordiale e fruttuoso rapporto di collaborazione intellettuale e soprattutto poetica tra il canonico di Barbana, Pietro Stancovich ${ }^{1}$ e il dignanese Martino Fioranti, ${ }^{2}$ esistono, tra i documenti custoditi presso l'archivio

1 Pietro Stancovich (Barbana 1771-1852), dopo aver compiuto studi di filosofia e teologia rispettivamente a Udine e a Padova, ritornò nel borgo istriano dove esercitò per tutta la vita la missione sacerdotale. Fu canonico e studioso eclettico e s'interessò con profonda dedizione ai più svariati campi disciplinari, tra cui l'archeologia, la storia, la letteratura, le tradizioni popolari, le scienze naturali quali la zoologia, la botanica, la geologia, non rifuggendo nemmeno dall'invenzione di apparecchiature da impiegare nella lavorazione in campo agricolo e industriale. Scrisse numerose opere, tra cui la più importante è la Biografia degli uomini distinti dell'Istria (1828-29), che lo consacrò quale "Plutarco istriano". Membro di varie Accademie italiane ed europee, fu tra gli intellettuali istriani più illustri della prima metà del XIX secolo, coltivando per tutta la vita amicizie e conoscenze con importanti intellettuali del suo tempo. Nel 1818 pubblicò pure una raccolta di poesie intitolata Versi.

2 Martino Fioranti, fu Andrea, nacque a Dignano tra il 12 e il 17 marzo 1795. Morì a Dignano il 24 luglio 1856 (vd. il Registro "Status animarum", 1853, accessibile per la consultazione su CD, "Vodnjan. Matične knjige" presso l'Archivio di Stato di Pisino). A stimolarci a consultare l'Archivio di Pisino fu principalmente una notizia rinvenuta nel libro di Domenico Rismondo, Dignano d'Istria nei ricordi (Società Tipografica Editrice, Bagnacavallo 1937, p. 101), in cui l'autore, elencando i vari podestà succedutisi nel governo della città, scrive a proposito del Fioranti: "12 ottobre 1853 - 21 giugno 1855: f. f. di podestà 
della Biblioteca universitaria di Pola, numerose attestazioni. Qui si tenterà di far luce, attingendo a quelle fonti, su un momento di particolare vivacità di tale sodalizio che vede impegnati, in un felice scambio poetico-epistolare, il canonico Stancovich e il dignanese Fioranti.

Redatti su due pagine di un unico foglio e custoditi nel fondo Fioranti, i documenti che ci accingiamo a esaminare rappresentano due poemetti occasionali: in quartine con versi a rima incatenata, di otto strofe più un distico finale quello scritto dal poeta di Dignano; in quartine a rima alternata, di nove strofe, invece, con anch'esso l'aggiunta di un distico in chiusura, quello redatto da Stancovich (rispettivamente figg. 1 e 2).

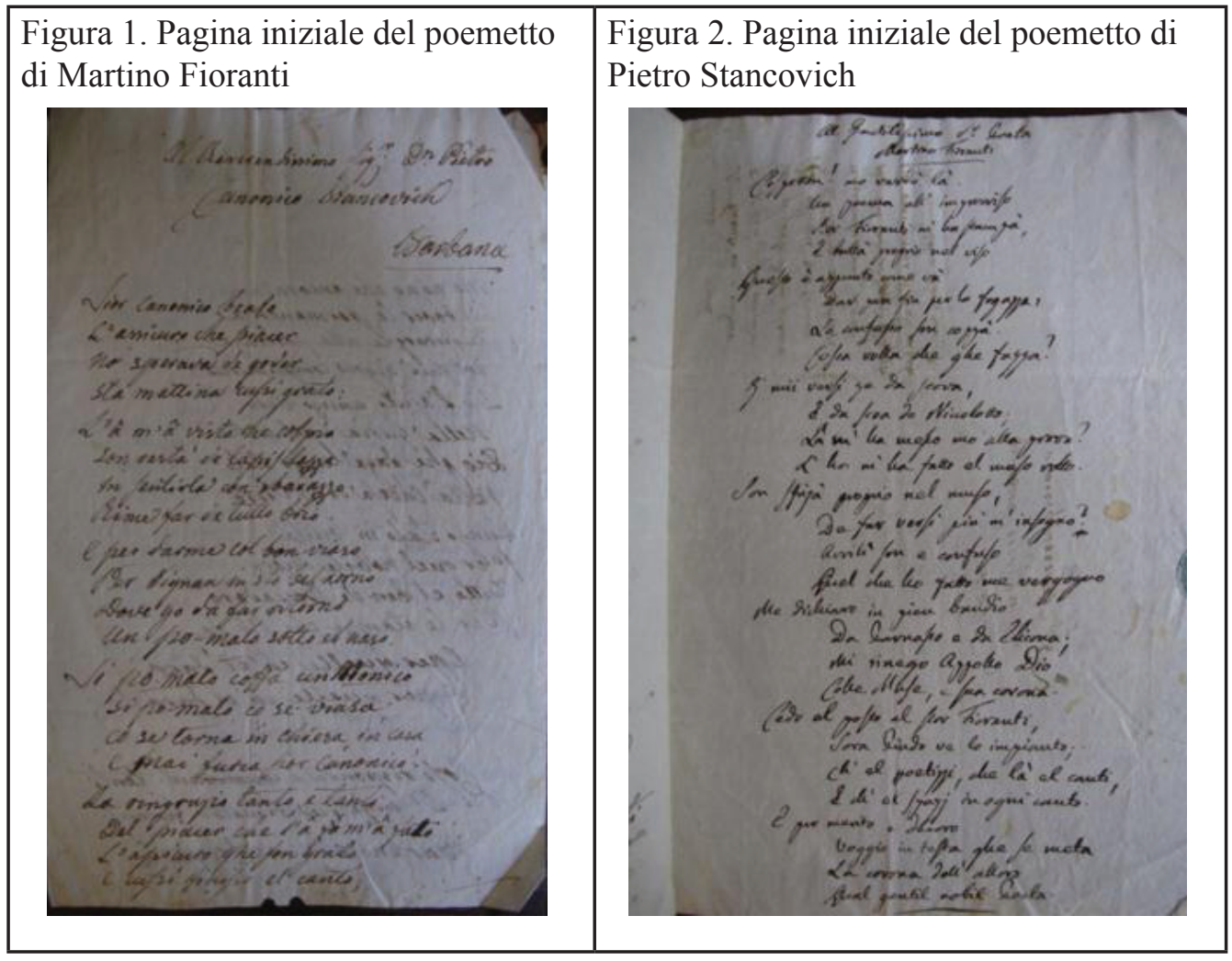

I due componimenti sono importanti perché, oltre a testimoniare un affettuoso rapporto di amicizia tra $\mathrm{i}$ due, in quello del Fioranti compare inoltre, in calce, la

Fioranti Martino. Si trovano tra le vecchie carte dei versi e dei sonetti di questo concittadino". Purtroppo, neppure in seguito a reiterate consultazioni della documentazione municipale di Dignano risalente a quel periodo, non abbiamo rinvenuto, presso l'Archivio di Pisino, alcun sonetto in dialetto dignanese. Da ciò potremmo desumere che i sonetti cui accenna il Rismondo siano quelli custoditi nel fondo "dalla Zonca" della Biblioteca Universitaria di Pola, tanto più che la grafia in cui sono scritti due sonetti (Zura un dagno de campagna e La spallareina dignagnisa defendo le siuritte) è identica alla grafia in cui sono redatti gli Atti comunali nel periodo in cui Martino Fioranti fu f. f. di podestà di Dignano, e cioè, a varie riprese, tra il 1818 e il 1855, e con la quale li abbiamo confrontati (vd. DAPA, 0043, Registar fondova, "Općina Vodnjan", Archivio di Stato di Pisino). 
data e il luogo di composizione: Barbana 4 xmbre 1826. Al poemetto del Fioranti, Stancovich risponde con un componimento riportato sulla seconda pagina dello stesso foglio, composto probabilmente lo stesso giorno dell'incontro tra i due, o di poco posteriore. Inoltre, essendo i due poemetti redatti in dialetto istroveneto con contaminazioni dall'italiano e dal croato, rappresentano un curioso esempio di quel particolare fenomeno che Pier Paolo Pasolini osserva nella poesia dialettale del nord Italia e che definisce "semidialettalità, ossia il particolare macaronico della poesia in dialetto". ${ }^{3}$ Pasolini spiegava tale fenomeno come conseguenza della particolare posizione geografica di una regione, in quel caso l'Emilia. A una simile, o forse ancor più pertinente motivazione, si presta anche la particolare posizione dell'Istria. Da sempre zona di confine e di passaggio, crogiuolo di contatti e contaminazioni, la regione si presenta, al pari dell'Emilia pasoliniana, quale luogo ideale per il manifestarsi di quel "carattere stradale", nell'accezione che il linguista austriaco, Friederich Schürr, usava nel descrivere la "propagazione dei fenomeni glottologici". ${ }^{4}$ Qui, tale propagazione si attua attraverso le varie inoculazioni e contaminazioni linguistiche che, traendole dai differenti codici, l'autore aggiunge al ceppo portante del dialetto istroveneto.

Anche se di mediocre valore estetico-letterario, i due componimenti risultano però di notevole importanza soprattutto in qualità di testimoni di un'occasionale visita e della breve permanenza del Fioranti nel villaggio di Barbana. In seguito al congedo dall'amico canonico, il dignanese compone il breve poemetto in quartine di ottonari a rima incrociata e variamente rimate, cui si aggiunge una coda di due versi, anch'essi ottonari, in rima con il primo e il quarto verso dell'ultima quartina.

2. Dai motivi chiaramente espressi nei due componimenti, si deduce senza difficoltà che fu per primo Martino Fioranti a rivolgersi all'amico canonico con un'epistola in versi. In questa, il motivo principale che muove il dignanese a scrivere allo Stancovich è, come spiega nella quinta strofa, di ringraziarlo "[...] tanto e tanto / Del piacer che l'â m'â fato / L'assicuro ghe son grato“. L'autore non si spinge più addentro nella specificazione del piacer, ma dalla prima quartina, però, veniamo a sapere di un precedente, di una circostanza, cioè, che nell'animo del mittente aveva suscitato grande gioia e piacere. Si trattava, come ci spiega l'autore nella seconda quartina, della contentezza da questi provata nel sentire, quella mattina stessa, il canonico "[...] con sbarazzo / Rime far de tutto brio". La strofa è importante per due motivi: innanzitutto perché rende, come in una nitida immagine, l'espressione stupefatta del Fioranti “[...] restà de lapis lazzo" di fronte alla bravura del canonico ad esprimersi in versi e, in secondo luogo, essa è la testimonianza della maestria stessa e della padronanza di Stancovich all'improvvisazione poetica, rivelando così, ancora una volta, il carattere eclettico di questa importante figura di intellettuale istriano del XIX secolo, colto, ingegnoso, portato sia alla meditazione scientifica sia all'estemporaneità poetica.

Nelle due quartine successive si assiste al motivo della partenza da Barbana e al viaggio che il poeta sta per intraprendere per far ritorno a Dignano. A livello retorico

3 Pasolini, P. P., Passione e ideologia, Garzanti, Milano, 1977, p. 181.

4 Ibidem. Friedrich Schürr (1888-1980), linguista austriaco, è considerato il maggiore studioso dei dialetti romagnoli. 
l'autore immette, adottando il costrutto dell'anafora, fugaci spunti dal croato. Notiamo infatti la reiterazione, in tre versi successivi, dell'avverbio pomalo (po-malo), cioè "piano", "lentamente"; ma è doveroso osservare che è soprattutto qui che si rivela tutta la limitatezza di questo poetare artificioso e pretestuoso, in cui i versi, quando esulano dalla pura enunciazione del fatto, scadono a riempitivo, immessi nella struttura strofica al solo scopo di rispettare la rima e sopperire all'andamento monotono e cantilenante del narrato poetico. È il caso, ad esempio, del primo e del quarto verso nella terza quartina, dove, per trovare un verso, e in particolare una parola in rima con viaso - ancora coerente con il motivo trattato -, l'autore non esita a correlarla con il verso assolutamente bizzarro "Un po-malo sotto el naso". È ancora il caso della quartina successiva in cui, oltre alla curiosa associazione "po-malo - mai furia" (curiosa innanzitutto dal versante psicolinguistico di associazioni semantiche entro codici diversi), si assiste all'estroso accostamento "coffa-Monico - furia - canonico", anch'esso costruito essenzialmente in ottemperanza alle convenzionali soluzioni metrico-foniche adottate. In Fioranti, quindi, non c'è un intento esteticamente letterario o, se c'è, esso è debole, velleitario, limitandosi il dignanese - e accontentandosi - a un verseggiare occasionale, dettato da contingenze esteriori, da circostanze o avvenimenti fortuiti e in cui l'io poetico rimane assopito, afono a qualsivoglia sussulto emotivo o psicologico, lontano dal maroso romantico che proprio in quegli anni trovava, anche in Istria, il suo primo fedele interprete nell'isolano, e contemporaneo, Pasquale Besenghi degli Ughi.

Sono quindi rime facili, quelle di cui si diletta il Fioranti, non elevandosi al di là della poesia dialettale, nell'accezione pancraziana di poesia che rimane circoscritta entro lo stretto ambito locale e municipale, limitata, nella sua scelta linguistica, a recepire il dialetto come una lingua inferiore o ad un codice non adatto ad esprimere sentimenti, emozioni che potessero trascendere la stretta cerchia dei destinatari.

Quella di Fioranti è una poesia conviviale, scabra nella sua espressività, legata com'è alla presentazione realistica e oggettiva della realtà e dell'occasione che la muove. Ciò è tanto più evidente nel poemetto dedicato all'amico Stancovich, in cui non vi sono dettagli né descrizioni paesaggistiche, gli aggettivi sono quasi aboliti, il monologo scorre lineare e continuo, di verso in verso, senza interruzione; il ritmo è pure costante, privo di alterazioni, l'unica alternanza è data dall'oscillare dell'accento tonico tra la terza e la settima sillaba, attribuendo così al componimento quella caratteristica curva melodica monocorde, tipica della litania.

\section{Al Reverendissimo Sig. ${ }^{r}$ D. ${ }^{n}$ Pietro Canonico Stancovich}

\section{Barbana}

Sior canonico beato

L'assicuro che piacer

No sperava de goder

Sta mattina cussì grato: 
L'â m'â visto che colpio

Son restà de lapislazzo

In sentirla con sbarazzo

Rime far de tutto brio.

E per darme col bon viaso

Per Dignan in sto bel xorno

Dove go da far ritorno

Un po-malo sotto el naso.

Si po-malo coffa un Monico

Si po-malo co se viasa,

Co se torna in chiesa, in casa

E mai furia sior canonico.

La ringrazio tanto e tanto

Del piacer che l'â m'â fato

L'assicuro ghe son grato,

E cussi finisso el canto;

Ma no no che ancora sento

De dover la permanenza

Augurarghe alla partenza

Col più degno complimento

Dio l'ajuti amigo vero,

Nella furia, e nel pomalo

Dio ghe daga lungo stalo

Nella chiesa de S. Piero

Lungo stalo in società

Per quel nobile decoro

Tutto el ben de Boccadoro

Per le stampe che l'â dà

E per quelle ch'el darà

E per quelle ch'el darà.

Così risponde a' complimenti tanti

L'infelice rimator Fioranti

Barbana 4 xmbre 1826 
3. La risposta di Stancovich testimonia anch'essa fedelmente l'occasionalità dei due componimenti. Infatti, L'infelice rimator Fioranti, come quest'ultimo si firma sotto al distico di coda, sembrerebbe aver scritto e consegnato il poemetto al canonico, o una minuta di questo, prima di accomiatarsi dall'amico o al momento stesso della partenza. A ciò rimandano i deittici temporali che troviamo sia nei versi di Fioranti (Sta mattina, sto bel xorno), sia in quelli di Stancovich (all' improvviso), nonché, in quest'ultimo, l'augurio di buon viaggio che rivolge all'amico.

Anche nel poemetto di Stancovich il tono è molto prosaico e familiare, volutamente scherzoso nel ritenersi l'autore, nei confronti dell'amico ritenuto miglior poeta, "[...] in pian bandio / Da Parnaso e da Elicona," ("confinato in pianura / Dal Parnaso e da Elicona"). Ma proprio questa familiarità con i luoghi e i personaggi del mondo classico (seguono riferimenti ad Apollo, alle Muse, al Pindo) rivela la versatilità dell'autore nella composizione letteraria, la conoscenza e la padronanza delle convenzioni metrico-stilistiche, come testimonia, tra l'altro, la già citata silloge arcadico-classicistica dei Versi, edita nel 1818.

$\mathrm{Al}$ di là del tono palesemente ironico che leggiamo nel poemetto stancovichiano, dal quale però traspare una disposizione di bonaria simpatia e fiduciosa aspettativa nei confronti del più giovane collega dignanese, non ci sembra esagerato supporre che quest'ultimo abbia goduto, perlomeno entro il ristretto ambito locale e tra i suoi contemporanei, di una certa nomea nell'arte della poesia. A ciò rimanderebbero $\mathrm{i}$ contatti, gli scambi epistolari che il Fioranti avrà sicuramente intrattenuto, oltre che con Stancovich, anche con altri personaggi abituati o interessati alle lettere. Va inoltre ancora ricordata a tale proposito, la rete di relazioni coltivata con chi trascrisse le sue poesie che, come Stancovich, ci ha lasciato, sul calco di quelle, redazioni nuove, corrette o appena ritoccate, ma sempre, però, aperte a una nuova lettura e a una nuova indagine della realtà che ci trasmettono, perché, come osservava acutamente Arnaldo di Benedetto, citando Cesare De Lollis

la poesia, e con lei la vita tutta dello spirito, [...] è come un coro che si prosegue nei secoli, e la nuova voce non può risonarvi come nuova se non ascoltando e accogliendo in sé le precedenti, e rispondendo ad esse, e ripigliando da esse il canto e continuandolo a suo e insieme a lor modo. ${ }^{5}$

In Stancovich quell'apprezzamento doveva essere certamente sincero, espressione non solo della sua affinità nei confronti del Fioranti come uomo, ma anche, al contempo, poeta. Già nel titolo del suo componimento, il canonico si rivolge all'amico chiamandolo Gentilissimo . $^{r}$ Poeta, intessendo, poi, nelle nove quartine a rima alternata, un vero e proprio panegirico che, anche se espresso in un tono scopertamente ironico, con continui sconfinamenti nel farsesco e nel comico, rimane però sempre ancorato a una comicità affabile, misurata, di bonario e innocente divertissement in rima. A prevalere, quindi, nel poemetto di Stancovich, a differenza di quello del Fioranti in cui spicca il motivo del ringraziamento con accenni moralistico-religiosi, è un'amorevole e bendisposta ironia. Non va però dimenticato neppure il motivo didattico, tipico anch'esso della poesia narrativa, e che il canonico qui usa con finezza d'intenti in

5 De Lollis, C., in di Benedetto, A., Poesia e critica del Novecento, Liguori, Napoli 1999, p. 164. 
termini parodistici, ironizzando cioè sulla propria competenza poetica per trasmettere invece all'amico e discepolo, efficacemente, l'esatto opposto: maestria ed inventiva d'immaginazione che forse rientravano tra quei "complimenti tanti" per i quali il Fioranti ringraziava il religioso in chiusura del suo poemetto. Ancora: mentre dai versi del Fioranti traspare un tono serio e ossequioso, da quelli di Stancovich a trasparire invece è un senso di ilare spensieratezza, è l'immagine di un festoso giocare con le parole, come il puer aeternus che costruisce e distrugge la propria realtà: "Lo confesso son coppà /[...] // Avvilì son e confuso / Quel che ho fatto me vergogno", per poi ricostruirla nuovamente in virtù della sua intrinseca capacità di rinnovarsi; ma anche quella, chiara, consigliera e lungimirante, del senex quando augura al suo allievo, nell'ultima quartina, "buon viaggio" - metafora della vita - da affrontare "a pian, pomallo".

È curioso osservare come le ultime due quartine del componimento del barbanese siano scritte in italiano, realizzando così compiutamente quella contaminazione tra lingua e dialetto cui si è già accennato e come riprenda qui, dai versi del Fioranti, per ripresentarlo nell'explicit al componimento, l'avverbio croato pomallo (< "pomalo", lentamente, piano) che, nella sua espressione fonica, suggerisce, in seguito all'esaurirsi dell'occlusiva $p$ e al reiterarsi della vocale oscura e chiusa $o$ tra le lievi e dolci $m$ e $l$, una lentezza, un appesantimento del moto, riuscendo così quasi a dare l'immagine sinestetica del procedere "a pian, pomallo".

Il poemetto stancovichiano, in risposta a quello dell'amico Fioranti, consta di nove quartine di ottonari a rima variamente alternata e, in chiusura, di un distico a rima baciata. Il testo, come trascritto sotto, si trova sulla terza facciata del foglio contenuto nel fondo Fioranti:

\section{Al Gentilissimo S.r Poeta Martino Fioranti}

Cospetton! Ma vardè là.

Un poema all'improvviso

Sior Fioranti mi ha stampà,

E buttà proprio nel viso

Questo è appunto come va

Dar pan fin per la fogazza!

Lo confesso son coppà.

Cossa volla che ghe fazza?

I mii versi ze da scova,

E da scoa de Nicolotto;

La m' ha messo no alla prova?

$L$ ' ha m'ha fatto el muso rotto.

Son sfrigà proprio nel muso,

Da far versi più m' insogno?

Avvilì son e confuso

Quel che ho fatto me vergogno 
Me dichiaro in pian bandio

Da Parnaso e da Elicona;

Mi rinego Appollo Dio

Colle Muse e sua corona.

Cedo el posto al sior Fioranti,

Sora Pindo ve lo impianto;

Ch'el poetizzi, che là el canti,

Ech'el spazi in ogni canto.

E per merito e decoro

Voggio in testa ghe se meta

La corona dell'alloro

Qual gentil nobil Poeta.

Sulla quarta facciata, dove si legge anche l'indirizzo del ricevente, cioè " $A l$ Reverendissimo sig. Don Pietro Stancovich. Barbana", Stancovich scrive ancora dieci versi:

Son venuto per sapere

Se Fioranti va a vedere

I suoi figli, e sua mogliera

Per pregarlo di un piacere

Nel volermi far tenere

Delle lettere al postiere.

Augurando buon viaggio

Ma che vadi addaggio addaggio,

Né mai ponga il piede in fallo

Ma cammini a pian, pomallo.

4. In questo lavoro si è esaminato uno tra i quattro componimenti di sicura attribuzione a Martino Fioranti, rinvenuti nell'archivio della Biblioteca universitaria di Pola: Al Reverendissimo Sig. ${ }^{r}$ D. ${ }^{n}$ Pietro Canonico Stancovich. Gli altri tre sonetti sono: Sul piccato, Xura un dagno de campagna, La spallareina dignagnisa.

Tutti i testi sono accomunati dalla particolare disposizione del poeta a trasporre in versi una sensibilità che trova principale alimento nella celebrazione della piccola patria municipale e dell'evento occasionale. Il carattere, quindi, folcloristico ( $L a$ spallareina) e, soprattutto, paesano (Xura un dagno de campagna, La spallareina, ma elementi ne troviamo anche nel poemetto al Reverendissimo) trattengono il linguaggio poetico fiorantiano a quella che Pier Vincenzo Mengaldo definisce "lingua della realtà", antitetica ed estranea a quella che, soltanto in seguito alla svolta primonovecentesca, perverrà a "lingua della poesia". ${ }^{6}$ Ecco, allora, che fatti e avvenimenti della piccola

6 Chiesa, M., Appunti sullo studio della poesia contemporanea in dialetto, in P. Mazzamuto (a cura di), La letteratura dialettale in Italia, "Annali della Facoltà di lettere e filosofia dell'Università di Palermo", Palermo, 1984, p. 873. 
cronaca locale divengono, per il nostro poeta, argomento privilegiato della sua esperienza poetica, da analizzare e svolgere con i modelli propri della sensibilità tardo arcadica e ingenuamente realista del secondo Settecento, e che si presenterà con code e strascichi ancora lungo tutto l'Ottocento. Sensibilità, questa, $\mathrm{o}$, più correttamente, dimensione storica e culturale alla quale il Fioranti appartiene interamente, discosto com'era - si è visto - dal subbuglio romantico che in quegli anni stava appena lambendo la realtà letteraria istriana, ma solo, però, sul piano della lingua letteraria standard.

Possiamo pertanto osservare, tra gli stilemi ricorrenti di tale modalità espressiva nel Fioranti, l'incipit sempre allocutivo dei suoi componimenti: Compàro Pridigadùr mei i vo seintù (Sul piccato), Ven zà, ven zà che te favelli un po (Xura un dagno de campagna), Sior canonico beato (poemetto), ma anche nella Spallareina il mei poetico che si rivela al settimo verso è lo stesso che inizia la sua dichiarazione con Fusso quilui che jò impetà sui muri... Da qui, il discorso si fa sempre enunciazione di una realtà circoscritta e ben individuata: la predica, il danno, la canzonatura delle siuritte, la visita all'amico Stancovich, che il più delle volte, in armonia all'aspettativa dell'espressione dialettale, scade nella comicità ingenua e consueta della rappresentazione del personaggio-macchietta, o di una situazione tipicamente ridanciana o, ancora, nella raffigurazione, con tratteggi coloriti e umoristici, di momenti tratti dal vissuto semplice e quotidiano della cittadina istriana.

Un altro tratto che frequentemente ricorre nei componimenti di Fioranti è, sul piano dell'espressione, l'intenso impiego di deittici spazio-temporali che vincolano la poesia alla dimensione del particolare, del discorso cronachistico, trattenendola così da una più alta manifestazione dell'esperienza. L'unico scritto in cui, soltanto limitatamente, possiamo ravvisare un accenno a tale esperienza è il primo sonetto di Fioranti, Sul piccato, in cui assistiamo alla presentazione di un'esperienza in sé traumatizzante per un credente, quale la paura della perdizione eterna, accompagnata però dalla presenza straniante di elementi appartenenti alla più ingenua tradizione demonologica popolare che le tolgono, così, l'alone angoscioso a favore di una coloritura più espressamente comico-grottesca.

Concludendo, possiamo pertanto asserire che, con i versi di Fioranti, ci troviamo ancora lontani da quella che Pier Vincenzo Mengaldo ha definito "dialettalità introversa $\mathrm{o}[\ldots]$ 'endofasica' “, 7 peculiare soltanto della nuova, mutata sensibilità novecentesca, e corollario alla precedente esperienza postunitaria di una poesia in dialetto intesa come una "apertura comunicativa, e comunitaria". ${ }^{8}$

7 Mengaldo, P. V. (a cura di), Introduzione a Poeti italiani del Novecento, Oscar Mondadori, Milano, $2011^{4}$, p. LXXI.

8 Ibidem 


\section{BIBLIOGRAFIA}

CHIESA, M., 1984, Appunti sullo studio della poesia contemporanea in dialetto, in MAZZAMUTO, P., (a cura di), La letteratura dialettale in Italia, "Annali della Facoltà di lettere e filosofia dell'Università di Palermo", Palermo.

DI BENEDETTO, A., 1999, Poesia e critica del Novecento, Liguori, Napoli.

MENGALDO, P. V., 20014, (a cura di), Introduzione a Poeti italiani del Novecento, Oscar Mondadori, Milano.

PASOLINI, P. P., 1977, Passione e ideologia, Garzanti, Milano. 


\section{OPAŽANJA O RAZMJENI KRATKIH PJESAMA IZMEĐU PIETRA STANCOVICHA I MARTINA FIORANTIJA}

Ovaj rad prikazuje komparativnu analizu između dviju kratkih prigodnih pjesama na istromletačkom dijalektu jugoistočne Istre. Sastave su 1826. godine napisali Vodnjanac Martino Fioranti i barbanski kanonik Pietro Stancovich, a predstavljaju važno svjedočanstvo postojanja pjesničkoga stvaralaštva na onome što je svojevremeno bio koiné istarskoga područja, odnosno na idiomu neposrednoga mletačkog podrijetla.

Kratke pjesme nemaju osobiti književni značaj. Zanimljivije su ako se pak promatraju s povijesnoga i dokumentarističnoga stajališta, koji otkriva sredinu, duh vremena, a pogotovo odnos srdačnog prijateljstva koji je spajao pjesnike.

$\mathrm{Na}$ koncu se kratko uspoređuje kratka pjesma s trima sonetima Martina Fiorantija pronađenih u arhivu Sveučilišne knjižnice u Puli.

Ključne riječi: kratka pjesma, dijalekt, istromletački, Fioranti, Stancovich

\section{OBSERVATIONS ON THE EXCHANGE OF SHORT POEMS BETWEEN PIETRO STANCOVICH AND MARTINO FIORANTI}

The paper presents a comparative analysis of two short poems in the Istroveneto dialect of the south-west Istria. The poems, written in 1826 by Martino Fioranti from Vodnjan and clergyman Pietro Stancovich from Barban, represent an important evidence of the presence of poetic activity in, what was at the time, the koiné of the Istrian territory, i.e. an idiom of a direct Venetian origin.

The poems are of little literary importance. They seem more interesting, however, when viewed from a historical and documentary perspective, thus revealing the environment, the customs of the era and, most of all, a warm friendship between the two poets.

In the conclusion, the paper brings a brief comparison between a poem and three sonnets by Martino Fioranti, found in the archives of the University Library of Pula.

Keywords: short poem, dialect, Istroveneto, Fioranti, Stancovich 